\title{
Abundância e distribuição de Aeglidae (Crustacea: Anomura) em função do uso da terra na bacia hidrográfica do Rio Jacutinga, Rio Grande do Sul, Brasil
}

\author{
André Trevisan 1; Luiz Ubiratan Hepp ${ }^{2} \&$ Sandro Santos 1, 3 \\ 1 Laboratório de Carcinologia, Universidade Federal de Santa Maria. Avenida Roraima 1000, Cidade Universitária, Camobi, \\ 97105-900 Santa Maria, Rio Grande do Sul, Brasil. \\ 2 Laboratório de Biomonitoramento, Departamento de Ciências Biológicas, Universidade Regional Integrada do Alto Uruguai \\ e das Missões. Avenida Sete de Setembro 1621, 99700-000 Erechim, Rio Grande do Sul, Brasil. \\ ${ }^{3}$ Corresponding author. E-mail: ssantos@smail.ufsm.br
}

\begin{abstract}
Abundance and distribution of Aeglidae (Crustacea: Anomura) in function of land use in Rio Jacutinga hydrographic basin, Rio Grande do Sul, Brazil. This study investigated the influence of land use and occupation on the density and distribution of aeglids in the hydrographic basin of the Jacutinga river (northern state of Rio Grande do Sul, Brazil). Sampling was carried out during the summer and winter of 2004, at 12 sites distributed in river spring, agriculture, grazing, and urban areas. Animals were collected using a Surber sampler. An analysis of variance (ANOVA) was conducted to evaluate differences in the density of species in each land use and sampling period. Biological data were classified using cluster analysis (Euclidian distance; UPGMA). A total of 226 aeglids were captured, including 167 (78.89\%) specimens of Aegla platensis Schmitt, 1942 and 59 (26.11\%) specimens of A. singularis Ringuelett, 1948. The aeglid distribution was associated with land use and occupation. In river spring and grazing areas, A. platensis was predominant, whereas in urban and agricultural areas $A$. singularis was the most prevalent species.
\end{abstract}

KEY WORDS. Aegla platensis; Aegla singularis; bioindicators.

No mundo todo, as práticas intensas de atividades agrícolas, pastoris e urbanas provocam a degradação de grandes extensões de mananciais hídricos. Essa degradação ambiental observada ao longo dos ecossistemas aquáticos continentais traz consigo não somente prejuízos à qualidade de água, mas também, prejudica consideravelmente as comunidades de invertebrados que habitam esses ambientes (WeTzeL 1993).

Alguns estudos têm relacionado a estrutura da comunidade de invertebrados aquáticos ao uso e ocupação do solo em diferentes bacias hidrográficas, sendo que grande parte desses estudos mostram que a comunidade de invertebrados aquáticos é uma boa indicadora de alterações hídricas provocadas por atividades urbanas, agrícolas e pastoris realizadas na região de entorno de corpos d'água em diferentes intensidades (BunN et al. 1999, Stewart et al. 2000, Roy et al. 2003, Braccia \& Voshell-JR 2006, Niyogi et al. 2007, Molozzi et al. 2007, Hepr \& SANTos 2008).

Resíduos gerados por atividades humanas e lançados nos ecossistemas aquáticos podem causar um significativo decréscimo nas espécies nativas (SMITH \& LAMP 2008). Essa redução está associada a alterações na concentração de compostos químicos da água dos quais a fauna é dependente, como por exemplo, oxigênio dissolvido, nutrientes (nitrogênio e fósforo), concentrações de metais, condutividade elétrica e pH (Buss et al. 2002, 2004, ZaLidis et al., 2002, Moreno et al. 2006, MACGREgor \& WarRen 2006, Rhea et al. 2006, Silveira et al. 2006, NiYogi et al. 2007).
Certos tipos de atividades, como as agrícolas, além dos resíduos químicos lançados na água, podem gerar a eliminação da mata ripária de rios e riachos. Esta ação favoreceria o assoreamento das margens e conseqüentemente o aporte de sedimento para o leito destes corpos de água. Como conseqüência pode ocorrer uma diminuição da disponibilidade de micro-habitat do ambiente, o que resultaria no comprometimento da abundância e distribuição dos organismos.

Em alguns ambientes aquáticos do sul e sudeste do Brasil registra-se a presença de um grupo peculiar de crustáceos, os eglídeos. Estes são os únicos anomuros atuais vivendo em águas continentais (BOND-BUCKUP 2003). São organismos que vivem em rios, riachos, lagos e rios de cavernas, embaixo de pedras ou no folhiço submerso. Eglideos são considerados elos importantes na cadeia trófica dos ecossistemas aquáticos, pois servem de alimento para peixes, aves, rãs e jacarés, além de se alimentarem de detritos foliares e insetos aquáticos imaturos, sendo controladores dessas populações (AREnas 1976, Magni \& Py-Daniel 1989, Bueno \& Bond-Buckup 2004, Santos et al. 2008).

Estes crustáceos são encontrados apenas em ambientes com boas condições ambientais e águas relativamente bem oxigenadas, portanto, são extremamente sensíveis às variações ou perturbações ambientais, sendo que, quando estas ocorrem, podem levar a redução ou ao desaparecimento das populações (BOND-BuCKuP \& SANTOS 2007). BÜCKER et al. (2008), investigando

(c) 2009 Sociedade Brasileira de Zoologia | www.sbzoologia.org.br | All rights reserved. 
a distribuição destes organismos em ambiente natural, com boas condições ambientais, registraram que espécies simpátricas ocupam micro-ambientes principalmente em função da disponibilidade de alimento.

O objetivo desse estudo é avaliar a existência de relação entre a densidade de eglídeos e o uso da terra ao longo da bacia hidrográfica do Rio Jacutinga, região com destacado potencial agrícola no norte do estado do Rio Grande do Sul.

\section{MATERIAL E MÉTODOS}

Situado na Região Alto Uruguai do Rio Grande do Sul, o município de Jacutinga possui uma área total de $179 \mathrm{Km}^{2}\left(27^{\circ} 40^{\prime}\right.$ $52^{\prime \prime}-27^{\circ} 53^{\prime} 07^{\prime \prime}$ S e $52^{\circ} 38^{\prime} 48^{\prime \prime}-52^{\circ} 27^{\prime} 23^{\prime \prime} \mathrm{W}$, Fig. 1). A altitude média é de $650 \mathrm{~m}$, clima subtropical, com temperaturas médias anuais de $18^{\circ} \mathrm{C}$ e precipitação média anual de $2.300 \mathrm{~mm}$ (SCARIOT $\&$ ZANIN 2005). A economia é concentrada na agricultura (cerca de $55 \%$ da área do município), além de pastagens, cerca de $30 \%$ da área (SCARIOT \& ZANIN 2005). A bacia hidrográfica do Rio Jacutinga possui área de aproximadamente $56,6 \mathrm{~km}^{2}$, localizada totalmente na área do município. Possui cobertura de vegetação nativa de apenas 5\% da área do município. Os maiores fragmentos vegetais situam-se em regiões com declives acentuados onde a atividade agrícola mecanizada é impossibilitada. A bacia hidrográfica apresenta trechos de primeira a terceira ordens, com profundidades que variam de 0,05 e 0,40 $\mathrm{m}$ e vazões entre 1,58 e $2,70 \mathrm{~m}^{-3} \mathrm{~s}^{-1}$.

Duas campanhas amostrais foram realizadas nos períodos de verão (janeiro) e inverno (julho) de 2004 para coleta de amostras de água e dos indivíduos em 12 pontos distribuídos ao longo da bacia hidrográfica (Fig. 1), contemplando diferentes usos da terra observados (nascente, agricultura, pastagens e urbanas). A caracterização morfológica dos pontos de amostragem pode ser visualizada na tabela I. Para cada uso e ocupação da terra foram definidos três pontos de coleta considerando, sempre que possível, a homogeneidade morfológica dos mesmos. Os organismos foram coletados com um amostrador tipo Surber, com área de $0,1 \mathrm{~m}^{2}$ e rede de malha $250 \mu \mathrm{m}$. Em cada ponto de coleta, foram realizadas 10 pseudo-réplicas, totalizando $1 \mathrm{~m}^{2}$ de área amostrada por ponto. O material coletado foi fixado em campo com formalina $10 \%$ e conduzido ao laboratório para triagem em peneiras de malhas 2,0; 1,0; 0,5 e 0,25 mm, para posterior identificação de acordo com Bond-Buckup \& Buckup (1994). A abundância de eglídeos foi analisada em função da densidade de animais nas áreas de estudo $\left(\mathrm{N}\right.$ ind. $\left./ \mathrm{m}^{2}\right)$.

Em campo, a temperatura da água e o oxigênio dissolvido (OD) foram mensurados com um Oxímetro YSI-55, a condutividade elétrica foi mensurada com o auxílio de um condutivímetro LUTRON e o $\mathrm{pH}$ foi medido com um pHmetro JENCO. No laboratório foram quantificados alguns parâmetros físico-químicos da água: turbidez, íon amônia e fósforo total. Todos os métodos utilizados estão de acordo com os procedimentos metodológicos descritos em Standard Methods (APHA 1998).

Para avaliar as diferenças entre as variáveis ambientais em função dos diferentes usos da terra e diferentes períodos de coleta e a diferença entre as densidades de eglídeos em função dos diferentes usos da terra foi utilizada uma ANOVA, $\mathrm{p}<0,05$ (Gotelli \& Eluison 2004). A matriz de dados biológicos foi previamente transformada $(\log [\mathrm{x}+1])$ a fim de assumir condições de normalidade e homocedasticidade dos dados. Os dados biológicos foram classificados pela aplicação de uma Análise de Agrupamentos a partir de uma matriz de semelhança de distância Euclidiana entre unidades amostrais, utilizando o método de ligação por média não ponderada (UPGMA). Para avaliar a significância da nitidez de grupos formados na análise de agrupamentos foi empregado um teste baseado em auto-

Tabela I. Características morfológicas dos locais de amostragem na bacia hidrográfica do Rio Jacutinga.

\begin{tabular}{cllccc}
\hline Locais & Uso da terra & $\begin{array}{c}\text { Coordenadas } \\
\text { Geográficas (UTM) }\end{array}$ & Altitude (m) & $\begin{array}{c}\text { Profundidade (m) } \\
\text { (verão-inverno) }\end{array}$ & $\begin{array}{c}\text { Velocidade de correnteza (m s }{ }^{-1} \text { ) } \\
\text { (verão-inverno) }\end{array}$ \\
\hline H1 & Nascentes & $0350750 / 6927177$ & 635 & $0,28-0,21$ & $0,50-0,41$ \\
H2 & Nascentes & $0349570 / 6926168$ & 764 & $0,06-0,05$ & $0,29-0,52$ \\
H3 & Nascentes & $0348912 / 6931095$ & 545 & $0,09-0,26$ & $0,25-0,28$ \\
A1 & Agricultura & $0349496 / 6927772$ & 596 & $0,14-0,14$ & $0,84-0,51$ \\
A2 & Agricultura & $0351284 / 6931219$ & 619 & $0,05-0,06$ & $0,11-0,17$ \\
A3 & Agricultura & $0348208 / 6933828$ & 554 & $0,25-0,27$ & $0,90-0,88$ \\
P1 & Pastagem & $0346917 / 6929128$ & 657 & $0,05-0,05$ & $0,53-0,47$ \\
P2 & Pastagem & $0349242 / 6929111$ & 576 & $0,16-0,12$ & $0,75-0,90$ \\
P3 & Pastagem & $0346460 / 6930311$ & 641 & $0,03-0,08$ & $0,38-0,48$ \\
U1 & Urbana & $0348919 / 6931450$ & 543 & $0,24-0,33$ & $0,86-0,95$ \\
U2 & Urbana & $0664630 / 6898581$ & 639 & $0,09-0,13$ & $0,74-0,48$ \\
U3 & Urbana & $0348995 / 6932855$ & 547 & $0,17-0,19$ & $0,70-0,62$ \\
\hline
\end{tabular}




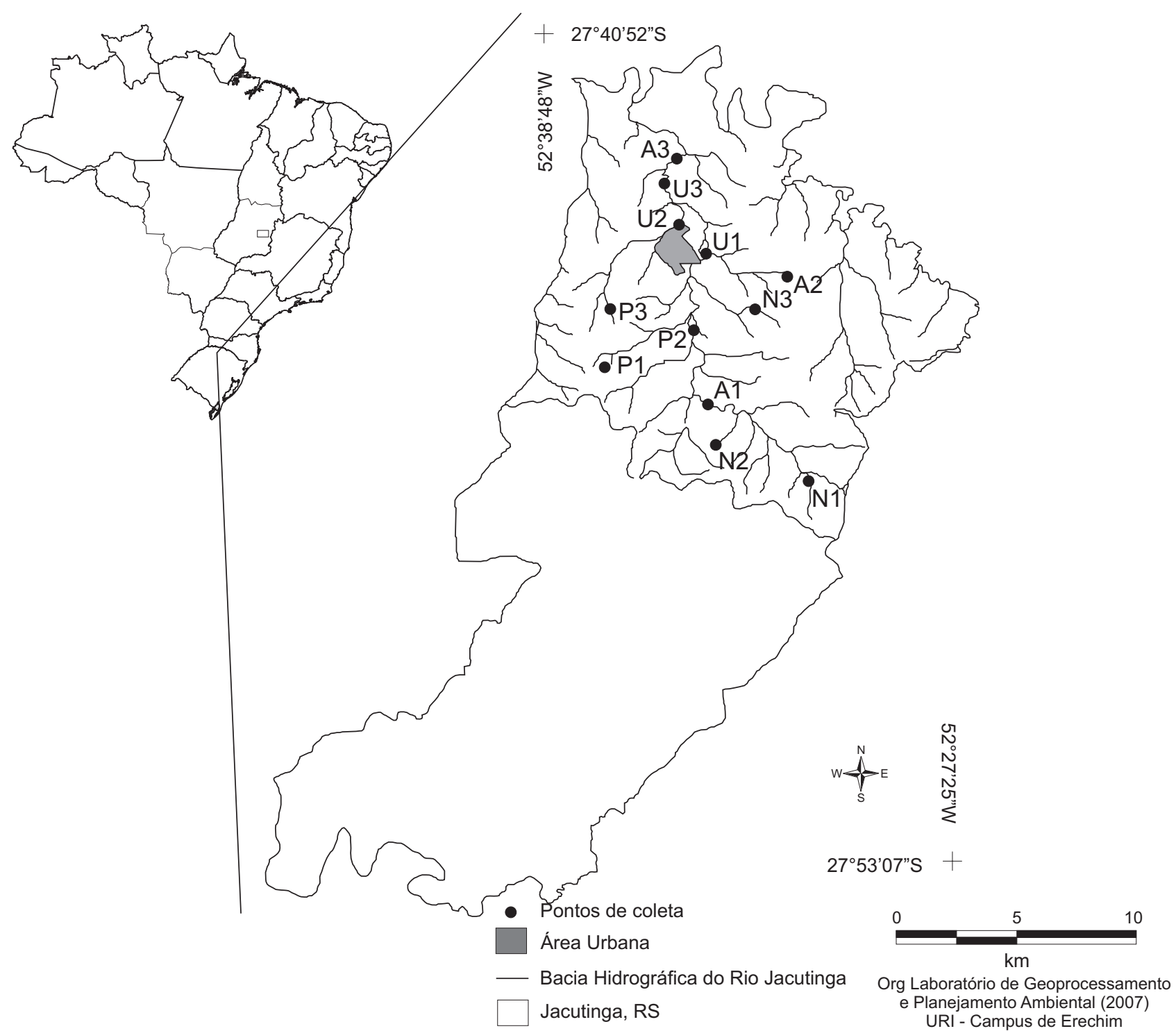

Figura 1. Localização da bacia hidrográfica do Rio Jacutinga e locais de coleta no município de Jacutinga, Rio Grande do Sul.

reamostragem (bootstrap) com 1000 interações usando um limiar de probabilidade $( \pm)$ de 0,05 (Pillar 1999). Este teste foi baseado em medidas de dissimilaridade entre cada par de unidades amostrais. O mesmo procedimento de classificação e avaliação da nitidez dos grupos foi utilizado para os dados das variáveis ambientais, no entanto a matriz de semelhança foi obtida através do índice de Gower entre unidades amostrais, sendo os dados previamente transformados pela extração da raiz quadrada dos valores de cada variável.

Os procedimentos estatísticos foram realizados utilizando o Software R (R Development Core Team 2006), Multiv 2.4.2 (disponível em http://ecoqua.ecologia.ufrgs.br) e MVSP 3.1 (Kovach Computing Services 2000).

\section{RESULTADOS}

Dentre as variáveis ambientais avaliadas, temperatura, turbidez, pH e fósforo total apresentaram diferenças significativas entre as estações do ano estudadas $(\mathrm{p}<0,05)$. A temperatura, $\mathrm{pH}$ e fósforo foram mais elevados no verão, enquanto turbidez apresentou maiores valores no inverno. Os demais parâmetros foram estatisticamente similares entre as estações ( $p>0,05$, Tab. II). A condutividade elétrica aumentou da nascente em sentido à foz. O oxigênio dissolvido apresentou comportamento inverso, com as menores concentrações sendo registradas na foz do rio $\left(5,26 \pm 2,5 \mathrm{mg} \mathrm{l}^{-1}\right)$. Os maiores valores de DBO foram encontrados próximos ao perímetro urbano. 
Somente amônia apresentou diferença significativa quando analisado em escala espacial, considerando os diferentes usos da terra $(p<0,05)$, sendo os valores médios desses parâmetros mais elevados nas áreas urbanas, pastoris e agrícolas (Tab. II). A análise de agrupamentos demonstrou a inexistência de similaridade entre as variáveis ambientais em relação ao diferentes usos da terra. No entanto foi observada a formação de dois grupos nítidos em função das estações do ano estudadas (Figs 2 e 3).
Foram amostrados, nos dois períodos de coletas, 226 eglídeos, sendo 167 exemplares de A. platensis Schmitt, 1942 (73,89\%) e 59 de A. singularis Ringuelett, 1948 (26,11\%). Aegla platensis foi observada em locais de nascentes e em trechos sob influência de pastagens, apresentando abundâncias de 12,6 e 14,4 ind $/ \mathrm{m}^{2}$, respectivamente. Aegla singularis foi coletada em locais com influência de todos os usos da terra, sendo observadas densidades de 1,$6 ; 7,6 ; 0,8$ e $1,8 \mathrm{ind} / \mathrm{m}^{2}$ em nascentes, áreas agrícolas, pastagens e áreas urbanizadas, respectivamente (Fig. 4).
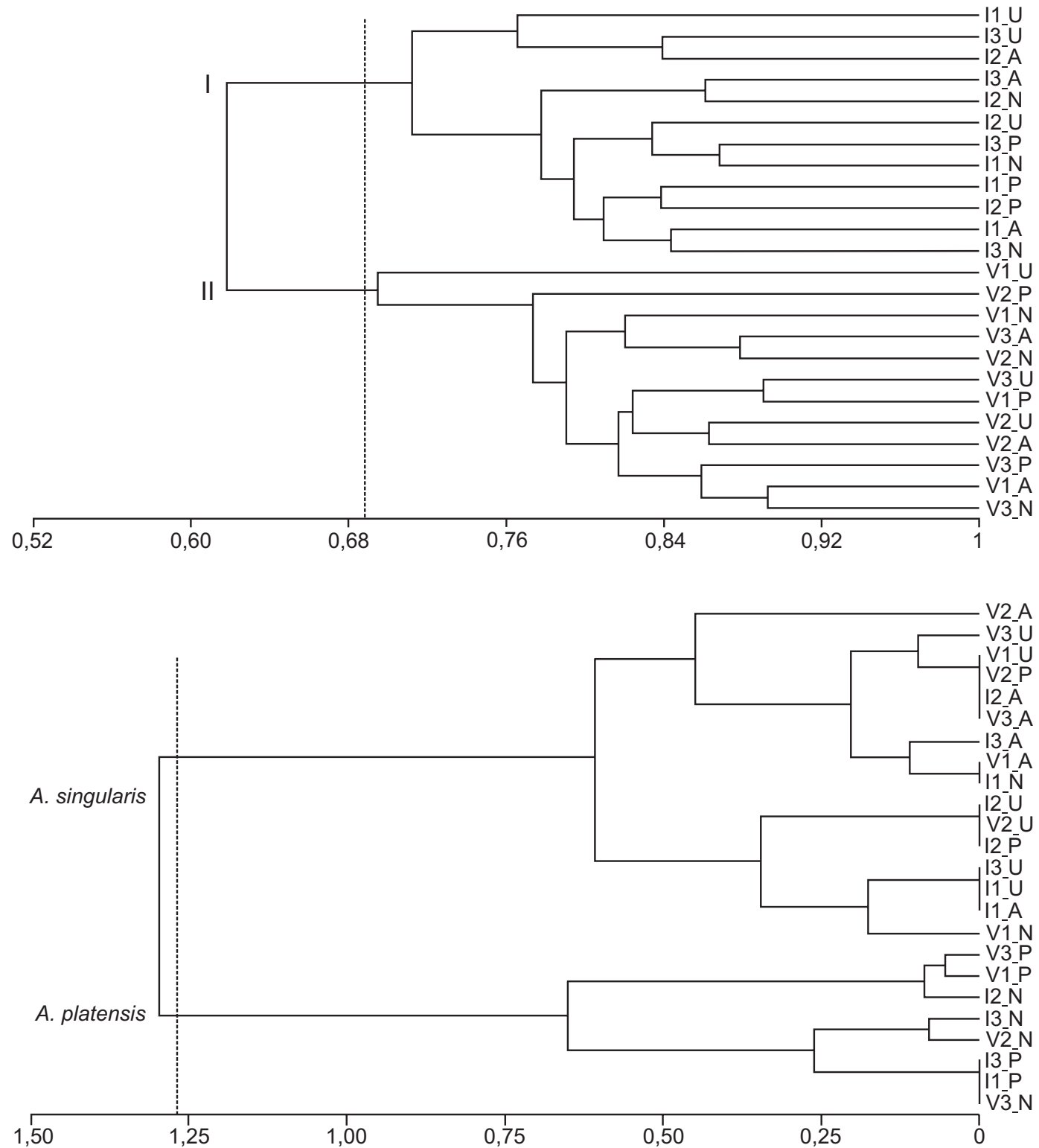

Figuras 2-3. Dendrogramas de (2) similaridade das variáveis ambientais (nível de agrupamento =0,69) e (3) abundância das espécies de Aegla em função do uso da terra (nível de agrupamento $=1,28$ ) na bacia hidrográfica do Rio Jacutinga, Rio Grande do Sul. 
Tabela II. Valores médios e desvio padrão dos parâmetros abióticos mensurados na bacia hidrográfica do Rio Jacutinga (Jacutinga, Rio Grande do Sul) em locais com diferentes usos do solo durante o verão e inverno de 2004.

\begin{tabular}{|c|c|c|c|c|c|c|c|c|}
\hline \multirow{3}{*}{ Variáveis } & \multicolumn{8}{|c|}{ Usos da terra } \\
\hline & \multicolumn{2}{|c|}{ Nascentes } & \multicolumn{2}{|c|}{ Agricultura } & \multicolumn{2}{|c|}{ Pastagem } & \multicolumn{2}{|c|}{ Urbana } \\
\hline & Verão & Inverno & Verão & Inverno & Verão & Inverno & Verão & Inverno \\
\hline Temperatura $\left({ }^{\circ} \mathrm{C}\right)$ & $21,2 \pm 2,3$ & $15,8 \pm 2,1$ & $20,9 \pm 0,8$ & $16,6 \pm 0,9$ & $22,3 \pm 1,5$ & $17,1 \pm 0,9$ & $23,5 \pm 1,6$ & $17,7 \pm 1,5$ \\
\hline Condutividade & $56,3 \pm 16,3$ & $56,8 \pm 14,1$ & $51,8 \pm 13,5$ & $57,3 \pm 12,7$ & $61,1 \pm 20,1$ & $58,6 \pm 9,3$ & $64,5 \pm 20$ & $70,8 \pm 3,4$ \\
\hline Turbidez (NTU) & $4,6 \pm 1,7$ & $6,4 \pm 2,4$ & $6,9 \pm 0,9$ & $8,3 \pm 3,3$ & $5,9 \pm 1,2$ & $13,2 \pm 4,2$ & $6,5 \pm 0,7$ & $10,3 \pm 1,2$ \\
\hline $\mathrm{pH}$ & $7,57 \pm 0,3$ & $6,85 \pm 0,4$ & $7,19 \pm 0,5$ & $6,85 \pm 0,87$ & $7,31 \pm 0,34$ & $6,51 \pm 0,21$ & $6,91 \pm 0,27$ & $6,04 \pm 0,24$ \\
\hline $\mathrm{OD}\left(\mathrm{mg} \mathrm{l}^{-1}\right)$ & $9,15 \pm 1,56$ & $8,70 \pm 0,53$ & $8,16 \pm 1,76$ & $6,93 \pm 3,34$ & $7,37 \pm 1,07$ & $7,40 \pm 1,90$ & $7,85 \pm 0,16$ & $5,26 \pm 2,46$ \\
\hline $\mathrm{DBO}\left(\mathrm{mg} \mathrm{l}^{-1}\right)$ & $4,8 \pm 1,85$ & $5,72 \pm 2,25$ & $4,44 \pm 0,95$ & $8,81 \pm 9,54$ & $3,79 \pm 1,13$ & $3,39 \pm 1,56$ & $5,0 \pm 1,05$ & $14,36 \pm 9,36$ \\
\hline Amônia (mg l-1) & $<0,001$ & $0,04 \pm 0,02$ & $0,01 \pm 0.001$ & $0,04 \pm 0,03$ & $0.013 \pm 0.02$ & $0.023 \pm 0.03$ & $0.07 \pm 0.04$ & $0.08 \pm 0.061$ \\
\hline Fósforo (mg l-1) & $0.13 \pm 0.116$ & $0.01 \pm 0.001$ & $0.184 \pm 0.05$ & $0.01 \pm 0.001$ & $0.122 \pm 0.09$ & $0.01 \pm 0.003$ & $0.456 \pm 0.64$ & $0.09 \pm 0.003$ \\
\hline
\end{tabular}

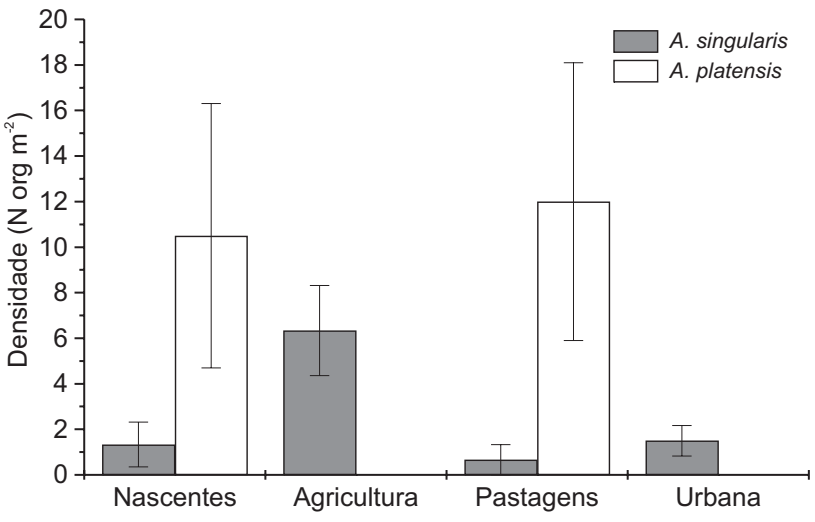

Figura 4. Densidade (ind $\mathrm{m}^{-2}$ ) de $A$. platensis e $A$. singularis em função do uso da terra na bacia hidrográfica do Rio Jacutinga, Rio Grande do Sul.

A presença geral de eglídeos, não considerando as particularidades de cada espécie, não foi influenciada pelos diferentes usos da terra observados ao longo da bacia do Rio Jacutinga ( $p>0,05$ ), visto que foram amostrados animais em áreas com influência de todos os usos de terra. No entanto, quando as espécies foram analisadas separadamente, observou-se que $A$. singularis possui sensibilidade quanto aos usos da terra $\left(\mathrm{F}_{3,20}=\right.$ $3,14 ; \mathrm{p}=0,04)$, sendo que as maiores diferenças foram observadas entre áreas agrícolas e com pastagens.

Aegla platensis também possui sensibilidade aos usos da terra, porém essa foi mais acentuada $\left(\mathrm{F}_{3,20}=5,28 ; \mathrm{p}=0,007\right)$. A principal diferença na densidade desta espécie ocorreu quando comparadas áreas de pastagens $\left(14,4 \mathrm{ind} / \mathrm{m}^{2}\right)$ com áreas agrícolas e urbanas (onde não foram amostrados indivíduos). Esse panorama é ratificado quando observado que $A$. platensis não ocorreu em áreas com uso agrícola e urbano, enquanto $A$. singularis foi observada em todos os tipos de ocupação da terra, com maior densidade em áreas agrícolas e urbanas. A. platensis apresenta maior densidade em regiões de nascentes. A densidade das espécies estudadas foi estatisticamente semelhante somente em áreas de nascentes ( $\mathrm{p}>0,05)$, sendo A. platensis levemente mais abundante. Em áreas agrícolas e urbanas $A$. singularis apresentou maiores densidades ( $\mathrm{p}=0,002$ e 0,02 , respectivamente), enquanto que em áreas sob influência de pastagens $A$. plantesis foi mais abundante $(\mathrm{p}=0,05)$.

A preferência das espécies por determinados usos da terra é corroborada pela análise de agrupamentos utilizando a abundância dos organismos (Figs 2 e 3). Na análise, observa-se a formação de dois grupos nítidos, sendo um mais basal, agrupando os organismos que ocorrem em nascentes e áreas de pastagens (A. platensis) e outro agrupando áreas agrícolas e urbanas, onde observa-se apenas a presença de $A$. singularis.

\section{DISCUSSÃO}

Além dos usos da terra nas regiões adjacentes aos rios, os parâmetros químicos da água são afetados pelas características geológicas e climáticas da região. Na bacia hidrográfica do Rio Jacutinga, as águas apresentam baixa concentração de íons dissolvidos, sendo que não foram observados valores maiores que $90 \mu \mathrm{Sm}^{-1}$ de condutividade elétrica. No entanto, a elevação na concentração da condutividade em regiões próximas a foz da bacia hidrográfica está associado ao aporte de matéria orgânica (LENAT \& CRAWFord 1994). Os parâmetros turbidez, DBO e fósforo total também aumentaram no sentido nascente - foz, sendo os maiores valores observados nos pontos mais próximos ao perímetro urbano, fato possivelmente associado com o aumento dos resíduos provenientes destas áreas (STEWART et al. 2000).

As variáveis oxigênio dissolvido e $\mathrm{pH}$ apresentaram resultados opostos aos observados nos demais parâmetros, sendo observado um decréscimo nos valores no mesmo gradiente. Esse fato está associado ao aumento da contaminação por matéria orgânica nas áreas não naturais, levando a um elevado 
consumo de oxigênio dos consumidores e uma redução do $\mathrm{pH}$ da água, devido a liberação de ácidos provenientes do processo de decomposição (Allan 1995, Brigante \& Espínola 2003, Trevisan \& Нерг 2007).

Ометто et al. (2004) citam que águas com elevados teores de amônia, geralmente provocados por resíduos urbanos, apresentam toxicidade que prejudicam a biota. No presente estudo foram observadas maiores concentrações dessa variável nas áreas próximas ao perímetro urbano; essa diferença é um indicativo de poluição pontual, visto que valores mais elevados desse íon estão associados a entradas de matéria orgânica, principalmente em áreas urbanas (STEWART et al. 2000, SALOMONI et al. 2007).

Com relação às variáveis ambientais e à presença de eglídeos em águas continentais, alguns trabalhos citam que teor elevado de oxigênio dissolvido na água é fundamental para o estabelecimento de populações de eglídeos, como se observa nos trabalhos de BAHAMONDE \& López (1961) com A. laevis Latreille, 1818, López (1965) com A. paulensis Schmitt, 1942, Rodrigues \& Hebling (1978) com A perobae Hebling \& Rodriguez, 1977, Bueno et al.(2000) com A. platensis, Swiech-Ayoub \& Masunari (2001) com A. castro Schmitt, 1942, e Colpo et al. (2005) com A. longirostri Bond-Buckup \& Buckup, 1994. Já a presença de eglídeos em águas com pH levemente ácidos ou alcalinos foi registrada por BuENO \& Bond-Buckup (2000), Noro \& Buckup (2002), Swiech-Ayoub \& Masunari (2001) e Gonçalves et al. (2006), em seus estudos com A. platensis, A. leptodactyla Buckup \& Rossi, 1977, A. castro e A. franciscana Buckup \& Rossi, 1977, respectivamente.

Não existem trabalhos publicados que abordem de que forma o uso e a ocupação da terra afetam as espécies de Aegla Leach, 1820. As poucas informações ecológicas sobre espécies do gênero não passam de pequenas referências dentro dos trabalhos de estrutura populacional, da mesma forma não existem relatos de como a química da água influencia a abundância de eglídeos em ambiente natural. Dentre as informações disponíveis destaca-se a preferência dos organismos por habitar substrato rochoso com a presença de folhiço proveniente da vegetação ripária. Swiech-Ayoub \& Masunari (2001) citam a presença de um grande número de $A$. castro Schmitt, 1942 em local com substrato arenoso, desprovido de pedregulhos ou cascalhos. BAHAMOnde \& López (1961) em seu trabalho com A. laevis Latreille, 1818, não observaram presença dessa espécie em substrato arenoso. Jovens e adultos de Aegla possuem uma tendência gregária e, de preferência, habitam locais sombreados e protegidos da forte correnteza como folhas submersas, troncos caídos, ou vegetação marginal (BAHAMONDE \& López 1961, LóPEZ 1965, Rodrigues \& Hebling 1978, Swiech-Ayoub \& Masunari 2001).

No presente estudo observa-se que as espécies de Aegla que ocorrem ao longo da bacia hidrográfica do Rio Jacutinga apresentam sensibilidade aos usos da terra. Aegla platensis mostrou-se uma espécie mais exigente, ocupando áreas com menor grau de impacto (nascentes e pastagens) e A. singularis, que, embora ocorra em todos os usos e apresente sensibilidade a estes, apresenta reflexos desse fato em sua estrutura populacional (densidades mais baixas). As atividades desenvolvidas ao longo da bacia hidrográfica não afetam de forma acentuada as características físicas e químicas da água, uma vez que se pode observar uma variação sazonal desses parâmetros. Assim, a distribuição de eglídeos ao longo da bacia hidrográfica do Rio Jacutinga pode estar relacionada com outros fatores ambientais relacionados ao uso e ocupação da terra tais como: cobertura ciliar, grau de erosão das margens, disponibilidade de alimento, diversidade de hábitats entre outros.

BÜCKER et al. (2008) citam que somente dois parâmetros relacionados à presença de matéria orgânica particulada grossa (de um total de 11 parâmetros abióticos) são determinantes para a abundância de A. itacolomiensis Bond-Buckup \& Buckup, 1994 e A. plantesis no Arroio Solitária Alta, no município de Igrejinha, Rio Grande do Sul. Bueno \& Bond-Buckup (2004) citam que fragmentos vegetais de origem terrestre são os itens mais importantes na alimentação das espécies $A$. plantesis e $A$. lingulata Bond-Buckup \& Buckup, 1994 no Arroio Mineiro no município de Taquara e em um tributário do Rio Tainhas em São Francisco de Paula, Rio Grande do Sul. A preferência de espécies de eglídeos por locais com presença de vegetação abundante também foi relatada por Castro-Souza \& Bond-Buckup (2004) em um tributário da bacia do Rio Pelotas no Rio Grande do Sul, sendo que a simpatria entre $A$. platensis e $A$. camargoi Buckup \& Rossi, 1977 é mais acentuada nesses ambientes. SANTos et al. (2008), em um trabalho que aborda a dieta natural de A. longirostri na região central do Estado do Rio Grande do Sul, citam que mesmo possuindo uma dieta variada esta espécie pode ser funcionalmente classificada como herbívora em virtude da predominância de tecidos vegetais em seu estômago, também enfatizando a importância da vegetação ripária como fonte de recursos para a comunidade bêntica em geral.

Além da distribuição e da abundância dos eglídeos ao longo da bacia hidrográfica do Rio Jacutinga ser influenciada pelo uso da terra e condições fisiográficas geradas por estes usos, aspectos referentes à competição interespecífica podem ter importância na distribuição destes organismos, e merecem ser tratados em estudos futuros. Se A. platensis apresentar uma maior capacidade de captar recursos ou for mais agressiva que $A$. singularis, os locais menos impactados (nascentes e áreas com pastagens) podem ser habitados preferencialmente por esta espécie.

O presente estudo é pioneiro no sentido de avaliar como o uso e a ocupação da terra influenciam a abundância de eglídeos. O conhecimento dessa relação é de fundamental importância para a criação de medidas conservacionistas, visto que os exemplares do gênero Aegla habitam rios de baixa ordem, sendo sensíveis a perturbações e/ou variações ambientais bruscas que podem levar a redução ou desaparecimento de populações. Dessa forma, a continuidade desses estudos é de fundamental importância para se determinar até que ponto o uso e a ocupação do solo são responsáveis pela presença ou 
ausência do gênero nos ecossistemas aquáticos continentais e até que ponto esse uso pode provocar alterações na estrutura das populações desses organismos.

\section{LITERATURA CITADA}

AlLAN, J.D. 1995. Stream Ecology. Structure and function of running waters. London, Chapman \& Hall.

АРнA. 1998. Standard methods for the examination of water and wastewater. Washington, American Public Health Association, AWWA, WEF, $20^{\text {th }}$ ed., 1162p.

ArenAs, R.L.1976. La cordillera de la costa como refugio de la fauna dulcícola preglacial. Archivos de Biología y Medicina Experimentales 10: 1-40.

Bahamonde, N. \& M.T. Lopez. 1961. Estudios biologicos en la populacion de Aegla laevis laevis (Latreille) de el Monte (Crustacea, Decapoda, Anomura). Investigaciones Zoológicas Chilenas 7: 19-58.

Braccia, A. \& J.R. Voshell-JR. 2006. Environmental factors accouting for benthic macroinvertebrate assemblage structure at the sample scale in streams subjected to a gradient of cattle grazing. Hydrobiologia 573: 55-73.

Brigante, J. \& E.L.G. SPíndola. 2003. Limnologia Fluvial: um estudo no Rio Mogi-Guaçu. São Carlos, RiMA, 255p.

BunN, S.E.; P.M. Davies \& T.D. Mosisch. 1999. Ecosystem measures of river health and their responses to riparian and catchment degradation. Freshwater Biology 41: 333-345.

Bond-Buckup, G. \& L. Buckup. 1994. A familia Aeglidae (Crustacea, Decapoda, Anomura). Archivos de Zoologia 2: 159-346.

Bond-Buckup, G. 2003. A família Aeglidae, p. 21-16 In. G.A.S. Melo (Ed.). Manual de identificação dos Crustacea Decapoda de água doce do Brasil. São Paulo, Editora Loyola, 429p.

Bueno, A.A.P. \& G. Bond-Buckup. 2000. Dinâmica populacional de Aegla platensis Schmitt (Crustacea, Decapoda, Aeglidae). Revista Brasileira de Zoologia 17: 43-49.

Bueno, A.A.P. \& G. Bond-Buckup. 2004. Natural diet of Aegla platensis and Aegla lingulata Bond-Buckup \& Buckup (Crustacea, Decapoda, Aeglidae) from Brazil. Acta Limnológica Brasiliensis 16 (2): 115-127.

Bueno, A.A.P.; G. Bond-Buckup \& L. Buckup. 2000. Crescimento de Aegla platensis em ambiente natural (Crustacea, Decapoda, Aeglidae). Revista Brasileira de Zoologia 17 (1): 43-49.

Bond-Buckup, G. \& S. SAntos. 2007. Crustáceos Anomuros de Águas Continentais: Diversidade e Aspectos Biológicos. Ciência e Ambiente 35: 47-54.

Bücker, F.; R. Gonçalves; G. Bond-Buckup \& A.S. Melo. 2008. Effects of environmental variables on the distribution of two freshwater crabs (Anomura: Aeglidae). Journal of Crustacean Biology 28 (2): 248-251.

Buss, D.F.; D.F. Baptista; M.P. Silveira; J.L. Nessimian \& L.F.M. DoRvillé. 2002. Influence of water chemistry and environmental degradation on macroinvertebrate assemblages in a river basin in south-east Brazil. Hydrobiologia 481: 125-136.
Buss, D. F.; D.F. Baptista; J.L. Nessimian \& M. EgLER. 2004. Substrate specificity, environmental degradation and distrubance structuring macroinvertebrate assemblages in neotropical streams. Hydrobiologia 518: 179-188.

Castro-Souza, T. \& G. Bond-Buckup. 2004. The trophic niche of two sympatric Aegla Leach species (Crustacea, Aeglidae) in a tributary of hydrographic basin of Pelotas River, Rio Grande do Sul Brazil. Revista Brasileira de Zoologia 21 (4): 805-813.

Colpo, K.D.; L.D. Ribeiro \& S. Santos. 2005. Population biology of the freshwater Anomura Aegla longirostri (Aeglidae) from South Braziliam streams. Journal of Crustacean Biology 25 (3): 495-499.

Gonçalves, R. S.; D.S. Castiglioni \& G. Bond-Buckup. 2006. Ecologia populacional de Aegla franciscana (Crustacea, Decapoda, Anomura) em São Francisco de Paula, RS, Brasil. Iheringia, Série Zoologia, 96 (1): 109-114.

Gotelli, N. J. \& A.M. Ellison. 2004. A primer of ecological statistics. Massachusetts, Sinauer Associates, 510p.

Hepp, L.U. \& S. SANTos. 2008. Benthic communities of streams related to different land uses in a hydrographic basin in southern Brazil. Environmental Monitorind and Assessment. [doi: 10.1007/s10661-008-0536-7]

Kovach Computing Services. 2000. Multivariate Statistical Package v. 3.11. Kovach compiting services, available online at: http://www.kovcomp.com

Lenat, D.R. \& J.K. Crawford. 1994. Effects of land use on water quality and aquatic biota of three North Carolina Piedmont streams. Hydrobiologia 294: 185-199.

Lopez, M.T. 1965. Estudios biologicos en Aegla odebrechtii paulensis, Schmitt (Crustacea, Decapoda, Anomura). Boletim de Zoologia da Faculdade de Filosofia, Ciencia e Letras 25: 301-314.

MacGregor, C.J. \& C.R. Warren. 2006. Adopting sustainable farm management pratices within a Nitrate Vulnerable Zone in Scotland: the view from the farm. Agriculture, Ecosystems and Environment 113: 108-119.

Magni, S.T. \& V. Py-Daniel. 1989. Aegla platensis Schmitt, 1942 (Decapoda, Anomura) um predador de imaturos de Simuliidae (Diptera, Culicomorpha). Revista de Saúde Pública 23: 258259.

Molozzi, J.; L.U. Hepp \& A.S. Dias. 2007. Influence of Rice Crop on the Benthic Community in Itajaí Valley (Santa Catarina, Brazil). Acta Limnologica Brasiliensia 19 (4): 383-392.

Moreno, J.L.; C. Navarro \& J.D. Las Heras. 2006. Abiotic ecotypes in south-central Spanish rivers: referente conditions and pollution. Environmental Pollution 143: 388-396.

Niyogi, D. K.; M. Koren; C.J. Arbuckle \& C.R. Townsend. 2007. Stream community along a catchment land-use gradient: subsidy-stress responses to pastoral development. Environmental Management 39: 213-225.

Noro, C.K. \& L. Buckup. 2002. Biologia reprodutiva e ecologia de Aegla leptodactyla (Crustacea, Anomura). Revista Brasileira de Zoologia 19 (4): 1063-1074. 
Ometto, J. P.; A. Gessner; L.A. Martinelli \& M.C. Bernardes. 2004. Macroinvertebrate community as indicator of land-use changes in tropical watersheds, southern Brazil. Ecohydrology \& Hydrobiology 4: 37-49.

Pillar, V.P. 1999. How sharp are classification? Ecology 80 (8): 2508-2516.

R Development Core Team. 2006. R: a language and environment for statistical computing. Vienna, R Foundation for Statistical Computing, ISBN 3-900051-07-0, available online at: http//www.R-project.org.

Rhea, D.T.; D.D. Harper; A.M. Farg \& W.G. Brumbaugh. 2006. Biomonitoring in the boulder river watershed, Montana, USA: metal concentration in biofilm and macroinvertebrates, and relation with macroinvertebrate assemblage. Environmetal Monitoring and Assessment 115: 381-393.

Rodrigues, W. \& N.J. Hebling. 1978. Estudos biológicos em Aegla perobae Hebling e Rodrigues, 1977 (Decapoda, Anomura). Rio de Janeiro: Revista Brasileira de Biologia 38 (2): 383-390.

Roy, A.H.; M.J. Rosemond; M.J. Paul \& J.B. Wallace. 2003. Stream macroinvertebrate response to catchment urbanization (Georgia, USA). Freshwater biology 48: 329-346.

SAlomoni, S.E.; O. Rocha \& E.H. Leite. 2007. Limnological characterization of Gravataí River, Rio Grande do Sul. Acta Limnologica Brasiliensia 19: 1-14.

Santos, S.; L. Ayres-Perez; R.C.F. Cardoso \& C.C. Sokolowicz. 2008. Natural diet of the freshwater anomuran Aegla longirostri (Crustacea, Anomura, Aeglidae). Journal of Natural History 42 (13-14): 1027-1037.

SCARiot, E.C. \& E.M. Zanin. 2005. Diagnóstico Ambiental do municipio de Jacutinga - RS. Perspectiva 105: 33-42.
Silveira, M. P.; D.F. Buss; J.L. Nessimian \& D.F. BAPTISTA. 2006. Spatial and temporal distribution of benthic macroinvertebrate in a southeastern brazilian river. Brazilian Journal of Biology 66: 623-632.

SMITH, R. F. \& W.O. Lamp. 2008. Comparasion of insect communities between adjacent headwater and main-stern stream in urban and rural watersheds. Journal of the North American Benthological Society 27: 161-175.

Stewart, P. M.; J.T. Butcher \& T.O. Swinford. 2000. Land use, habitat and water quality effects on macroinvertebrate communities in three watersheds of a Lake Michigan associated marsh system. Aquatic Ecosystem Health and Management 3: 179189.

Swiech-Ayoub, B.P. \& S. Masunari. 2001. Flutuações temporal e espacial de abundância e composição de tamanho de Aegla castro Schmitt (Crustacea, Anomura, Aeglidae) no Buraco do Padre, Ponta Grossa, Paraná, Brasil. Revista Brasileira de Zoologia 18 (3): 1003-1017.

Trevisan, A. \& L.U. Hepp. 2007. Dinâmica de componentes químicos vegetais e fauna associada ao processo de decomposição de espécies arbóreas em um riacho no norte do Rio Grande do Sul, Brasil. Neotropical Biology and Conservation 2 (1): $55-60$.

Wetzel, R.G. 1993. Limnologia. Lisboa, Fundação Calouste Gulbenkian, 1014p.

Zalidis, G.; S. Stamatiadis; V. Takavakoglou; K. Eskridge \& N. Misopolinos. 2002. Impacts of agricultural pratices on soil and water quality in Mediterranean region and proposed assessment methodology. Agriculture, Ecossystems and Environment 88: 137-146.

Submitted: 01.XII.2008; Accepted: 12.IX.2009.

Editorial responsibility: Neusa Hamada 